ESAIM: M2AN 49 (2015) 1047-1062

DOI: $10.1051 / \mathrm{m} 2 \mathrm{an} / 2015002$
ESAIM: Mathematical Modelling and Numerical Analysis

www.esaim-m2an.org

\title{
UNCERTAINTY QUANTIFICATION IN THE NUMERICAL SOLUTION OF COUPLED SYSTEMS BY INVOLUTIVE COMPLETION
}

\author{
Bijan Mohammadi ${ }^{1}$ And Jukka TuOmela ${ }^{1}$
}

\begin{abstract}
We address the issue of epistemic uncertainty quantification in the context of constrained differential systems. To illustrate our approach we have chosen a certain chromatographic adsorption model which is a coupled system of partial differential, ordinary differential and algebraic equations. The difficulty in solving this type of a system is that typically certain unknowns lack a natural time evolution equation. The standard approach in such cases is to devise specific numerical schemes which somehow try to take into account the implicit structure of the system. In our approach, we complete the system by finding the appropriate missing evolution equations. This makes the system overdetermined and more complicated in some way but, on the other hand, the completed system provides extra information useful for error estimation and uncertainty quantification. We will also show that reducing the epistemic uncertainties also leads to better estimations of aleatory uncertainties.
\end{abstract}

Mathematics Subject Classification. 58J05, 35J40, 35S15.

Received March 25, 2014. Revised January 9, 2015.

Published online June 30, 2015.

\section{INTRODUCTION}

In the field of Uncertainty quantification (UQ) the uncertanties are classified in epistemic (reducible) and aleatory (irreducible) categories $[1,4,6,9,20]$. There are two main approaches to estimate aleatory uncertainties. One can use either statistical techniques directly or derive new models including the stochastic features of the parameters. The methods in the latter class are usually intrusive [5,22]. An important issue with all these methods is their high computational complexity. To overcome this limitation one usually introduces low-order models $[15,17,18]$ which are then used for UQ instead of the initial high-fidelity models.

Epistemic uncertainties, on the other hand, can be reduced by improving the modeling or solution procedures and it is one of our objectives to show how this can be done in the context of constrained coupled differential systems. Unlike classical numerical errors, epistemic uncertainties concern both the continuous and discrete levels. They can be reduced improving either the models or their discretizations. This paper concerns both issues as an initial model is first augmented before discretization. More precisely, we aim at addressing the uncertainties related to the numerical solution of differential systems which have constraints or in other words which are overdetermined. We propose a systematic and constructive approach which produces new equations

Keywords and phrases. Overdetermined PDE, uncertainty quantification, sensitivity analysis, chromatographic adsorption, constrained coupled systems.

1 University Montpellier II, France. bijan.mohammadi@um2.fr 
whose solutions quantify the level of the uncertainties on the variables. It is based on a reformulation of the initial system by completing the system, i.e. adding appropriate differential consequences to the system. This means that we work on the continuous level before any discretization.

We expect the completed system to be more suitable for discretization because the constraints are then better taken into account. Hence typically some standard discretization method which gives poor results when applied to the original system will be accurate and robust when applied to the completed system. We can thus easily use generic commercial tools to solve our problem. Moreover our approach makes it easier to reach higher accuracy by increasing the accuracy of the discretization for a particular variable without being limited by possible compatibility issues of discretizations of different variables. For instance, we previously showed that the inf-sup condition (see e.g. [3]) for the Stokes problem disappears after our reformulation: the system stays stable even with higher order discretizations for the pressure than velocity [12].

In general our aim is to incorporate the new features obtained by completing the given system as upgrades of existing solvers. In this way the considerable effort required in developing a new code from scratch is avoided [13]. These techniques are not very well-known in the numerical simulation communities but hopefully the present paper will help to popularize them further.

We will illustrate our approach by analyzing a well-known model of packed-bed chromatography. The physical idea of this interesting model is that there are several substances in the ambient fluid which are adsorbed on the boundary of the domain of the flow. This leads to a system where there are convection diffusion equations, ordinary differential equations and nonlinear algebraic equations.

The challenge of solving this kind of the system comes from the algebraic part of the system. Previous studies have treated the algebraic part of the problem as constraints and devised special methods such that the constraints would be as well satisfied as possible, see $[10,11]$ and references therein. The drawback is that different systems may well require different methods. To address this difficulty we use our general methodology and differentiate the constraints to obtain new dynamic equations for the "remaining" or "algebraic" variables. Remarkably this will always give a (nonlinear) convection diffusion equation for any constraints. Hence we can write time evolution equations for all variables and use any standard method to solve them. Since we can monitor the constraints we can then correct the solution such that it stays on the constraint manifold.

We think that this is useful for epistemic uncertainty reduction of constrained systems because, unlike for the initial systems, all relevant constraints of the problem are explicitly included in the completed system. In this way the errors related to constraints can effectively be controlled and the computations thus become more stable because one does not need to worry about possible instabilities due to the violation of constraints.

From the completed system we derive a related system, called the augmented system, which contains some new variables. They play the role of local uncertainty estimators and require only a very low extra computational cost. These variables can also be estimated for the initial system, as well as for different discretizations and so they provide a natural framework to measure the evolution of the uncertainties in various cases.

To implement our approach we start with an existing program where the constraints are imposed by using the Newton's method. Typically there are problems of convergence. Then we modify the program taking into account the completed and augmented system that we derived from the initial system. In particular we use the dynamics of the new equations to initialize the Newton iterations. This improves greatly the convergence properties of the method.

We will also analyze the impact of our reformulation on aleatory uncertainties when the functioning physical parameters of the model are not known exactly, but their probability density function (PDF) is assumed to be known. In particular, resolving the constraints accurately has a clear impact on the prediction of the first and second order moments of the variables.

In order to quantify uncertanties it is very important to analyze how uncertanties propagate. We introduce small perturbations at the inlet boundary and study how they propagate through the domain. It is seen that, unlike the initial model, the augmented model damps high frequency components very fast, as theoretical considerations predicted. 
In Section 2 we recall the general background information about PDE systems which are needed in our formulation. In Section 3 we describe the general form of the model and study its structural properties. In Section 4 we introduce a particular model of 2 substances which was given in $[10,11]$ and formulate it according to our approach. In Section 5 we give the numerical results and discuss the impact of our formulation on the epistemic and aleatory uncertainties. In particular, we show that it is not always possible to separate these two sources of uncertainties.

\section{InVolutive, COMPlETED AND AUGMENTED SYSTEMS}

Let us first briefly outline our general approach to constrained systems. For more details we refer to $[2,7,8,12,13,16,19,21]$. Let us consider a system:

$$
\tilde{A} u=\tilde{f} .
$$

This is the physical model we start with. This initial model contains in our case partial differential, ordinary differential and algebraic equations. Now differentiating these equations we obtain new equations which we call differential consequences or integrability conditions of the initial system. Evidently if $u$ is a smooth solution then it will also satisfy all the differential consequences of the system.

One may say that by computing differential consequences one makes explicit some information of the system which is only implicit in the original system. In particular one can thus analyze some structural properties of the system which is not possible using the initial system. Now it is a deep theorem in formal theory of $P D E[2,16,19,21]$ that (under certain relatively mild conditions) it is sufficient to differentiate the system a finite number of times to obtain "full" structural information the system. The system thus obtained is called the involutive form of the given system.

Note in particular that in spite of the highly abstract nature of the theorem the implications of the theorem are quite constructive. To look for the involutive form one needs only to differentiate and eliminate variables which even in nonlinear case is fairly feasible using Gröbner basis techniques which are readily available in many computer algebra systems. It turns out that for the purposes of numerical computation it is sometimes convenient to use not the full involutive form of the system, but to add just some of the integrability conditions to the original system. Hence we will use the term completed system to indicate that we may not use the full involutive system.

In all cases completed systems have more equations than unknowns. One possible way to deal with this is to solve the system in the least square sense. Another approach which we advocate here is to make the system square again by introducing new variables which will also play the role of error and uncertainty estimators.

Let us denote by $A_{0}$ the operator obtained form $\tilde{A}$ by adding some appropriate differential consequences to it and consider the completed system:

$$
A_{0} u=f .
$$

The right hand side $f$ is obtained from $\tilde{f}$ in the process of computing the differential consequences. However, since $A_{0}$ is overdetermined and has more equations than unknowns there are usually no solutions if $f$ is chosen arbitrarily. Hence our problem (2.2) has no solutions unless $f$ satisfies some compatibility conditions. These conditions are given by a compatibility operator $A_{1}$ such that $A_{1} A_{0}=0$ and $(2.2)$ has a solution only if $A_{1} f=0$.

Since $A_{1}$ contains important information about our system we will use it in the numerical solution. Let us now introduce some function spaces $V_{i}$ such that $A_{i}: V_{i} \rightarrow V_{i+1}$ and consider the following complex:

$$
0 \longrightarrow V_{0} \stackrel{A_{0}}{\longrightarrow} V_{1} \stackrel{A_{1}}{\longrightarrow} V_{2} \longrightarrow 0 .
$$

In the ideal situation this complex is exact; i.e. $A_{0}$ is injective, $A_{1}$ is surjective and image $\left(A_{0}\right)=\operatorname{ker}\left(A_{1}\right)$. This suggests that we could decompose $V_{1}$ as:

$$
\operatorname{image}\left(A_{0}\right) \oplus \operatorname{image}\left(A_{1}^{T}\right)=V_{1},
$$


where $A_{1}^{T}$ is the formal transpose of $A_{1}$. This decomposition means that it is indeed possible to find some functional framework in which the combined operator $\left(A_{0}, A_{1}^{T}\right)$ is bijective. So instead of trying to solve the system (2.2) in some least square sense, we introduce an auxiliary variable $z$ and solve

$$
A_{0} y+A_{1}^{T} z=f .
$$

We call this system the augmented system. The augmented system is square.

In previous works, we have shown how to proceed with this approach for quite general coupled differential systems (see [12-14] for examples of applications on constrained physical systems such as the Stokes and NavierStokes equations and coupled systems for microfluidic ionic flows). In this work we will illustrate the approach with a chromatography model.

\section{Application to a Coupled Chromatography MOdel}

We consider a fluid flow where there are several substances whose concentrations interest us. The concentrations of the substances are supposed to be so small that the flow can be considered to be independent of them. Further it is realistic to suppose that the flow is one dimensional and even constant. We denote by $x$ the coordinate of the domain along the flow and by $v$ the velocity field.

Let $C^{j}$ be the concentration of the substance $j$ in the fluid phase, $S^{j}$ concentration in the solid phase and $E^{j}$ the equilibrium concentration. We suppose that there are $\ell$ substances and denote $C=\left(C^{1}, \ldots, C^{\ell}\right)$ and similarly for other variables.

First we have identical differential equations for the components of $C$ and $S$.

$$
\begin{aligned}
& C_{t}-d C_{x x}+v C_{x}+\alpha k(E-S)=0 \\
& S_{t}-k(E-S)=0 .
\end{aligned}
$$

Here $d, \alpha$ and $k$ are some positive constants. In what follows we assume that $v$ is constant. Hence we have simple convection diffusion equations for $C$ and ODE for $S$. To close the system we have in addition an algebraic equation

$$
g(E, C)=0, \quad g: \mathbb{R}^{2 \ell} \rightarrow \mathbb{R}^{\ell} .
$$

To get more information about the time evolution of $E$ we differentiate the above equation. In the following $\partial_{t}$ denotes the total derivative with respect to $t$ and $\partial_{E} g$ is the differential of $g$ with respect to variables $E$. The second differentials are denoted similarly. This gives

$$
\begin{aligned}
\partial_{t} g & =\partial_{E} g E_{t}+\partial_{C} g C_{t}=0 \\
\partial_{x} g & =\partial_{E} g E_{x}+\partial_{C} g C_{x}=0 \\
\partial_{x x} g & =\partial_{x}\left(\partial_{E} g E_{x}\right)+\partial_{C} g C_{x x}+\left(\partial_{E C} g\right)\left(E_{x}, C_{x}\right)+\left(\partial_{C C} g\right)\left(C_{x}, C_{x}\right)=0 .
\end{aligned}
$$

Using these and the original equations (3.1) we obtain an evolution equation for $E$ :

$$
\begin{aligned}
\partial_{E} g E_{t} & -d \partial_{x}\left(\partial_{E} g E_{x}\right)+v \partial_{E} g E_{x} \\
& -d\left(\partial_{E C} g\right)\left(E_{x}, C_{x}\right)-d\left(\partial_{C C} g\right)\left(C_{x}, C_{x}\right)-\alpha k \partial_{C} g(E-S)=0 .
\end{aligned}
$$

Note that in addition to smoothness we did not need to assume any particular property of $g$ to derive this equation. Of course this is highly nonlinear in general. However, we have the following necessary condition for the equation (3.3) to be reasonable.

Lemma 3.1. Suppose that $C$ and $S$ are known and $\partial_{E} g$ is invertible. Then the linearization of (3.3) is parabolic.

Proof. With the above hypothesis the linearized equation is of the form

$$
E_{t}-d E_{x x}+\text { lower order terms }=0 .
$$


The hypothesis that $\partial_{E} g$ is invertible is perfectly reasonable since this guarantees by implicit function theorem that the variables $E$ could actually be solved in terms of $C$ using the constraint equations (3.2). In other words if this hypothesis were not satisfied then that would cast some doubt on the validity of the model itself.

It is somewhat curious that otherwise the lemma is valid whatever the actual constraint map $g$ is. Even the diffusion constant is always the same for both $E$ and $C$.

Our new system can now be written as

$$
\begin{aligned}
& C_{t}-d C_{x x}+v C_{x}+\alpha k(E-S)=0 \\
& S_{t}-k(E-S)=0 \\
& \partial_{E} g E_{t}-d \partial_{x}\left(\partial_{E} g E_{x}\right)+v \partial_{E} g E_{x} \\
& -d\left(\partial_{E C} g\right)\left(E_{x}, C_{x}\right)-d\left(\partial_{C} g\right)\left(C_{x}, C_{x}\right)-\alpha k \partial_{C} g(E-S)=0 \\
& g(E, C)=0 .
\end{aligned}
$$

Let us finally denote $u=(C, S, E)$ and write the above system simply as $A_{0} u=0$. Let us then introduce the operator

$$
A_{1}=\left(-\partial_{C} g, 0,-1, \partial_{t}-d \partial_{x x}+v \partial_{x}\right) .
$$

This operator codes the calculations by which we found the new equation (3.3) and hence $A_{1} A_{0}=0$. We call $A_{1}$ the compatibility operator of $A_{0}$. Now using the same idea as in our previous papers $[12,14]$ we introduce the augmented system

$$
A_{0} u+A_{1}^{T} z=0
$$

where $z$ are new variables. Writing this out in full gives

$$
\begin{aligned}
& C_{t}-d C_{x x}+v C_{x}+\alpha k(E-S)-\left(\partial_{C} g\right)^{T} z=0 \\
& S_{t}-k(E-S)=0 \\
& \partial_{E} g E_{t}-d \partial_{x}\left(\partial_{E} g E_{x}\right)+v \partial_{E} g E_{x} \\
& -d\left(\partial_{E C} g\right)\left(E_{x}, C_{x}\right)-d\left(\partial_{C C} g\right)\left(C_{x}, C_{x}\right)-\alpha k \partial_{C} g(E-S)-z=0 \\
& z_{t}-d z_{x x}-v z_{x}+g(E, C)=0 .
\end{aligned}
$$

Note in particular that convection for $z$ is "backwards". For the exact solution $z$ would be identically zero so we can use it to quantify and analyze the numerical error. Note that formally $z$ is a solution of the equation

$$
A_{1} A_{1}^{T} z=z_{t t}-2 d z_{x x t}+d^{2} z_{x x x x}-v^{2} z_{x x}+\left(I+\partial_{C} g\left(\partial_{C} g\right)^{T}\right) z=0 .
$$

Now $I+\partial_{C} g\left(\partial_{C} g\right)^{T}$ is symmetric and positive definite so let us perform the linear stability analysis in the scalar case and consider

$$
z_{t t}-2 d z_{x x t}+d^{2} z_{x x x x}-v^{2} z_{x x}+b z=0
$$

where $b>0$. Looking for the plane waves one finds that there are solutions of the form

$$
z(x, t)=\exp \left(i\left(k x-\sqrt{b+v^{2} k^{2}} t\right)-d k^{2} t\right) .
$$

Hence the high frequency components are strongly damped but low frequency components can propagate. We might therefore expect similar behavior also for the augmented model. This would also imply that the physical model itself is quite robust with respect to small high frequency perturbations of the concentrations. 


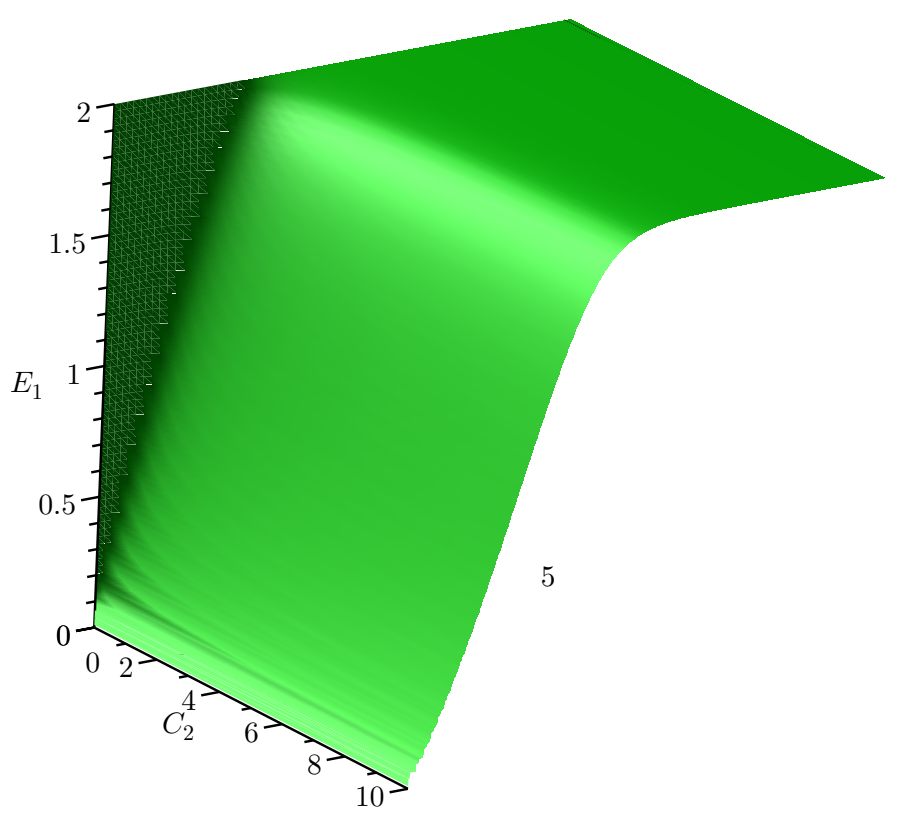

Figure 1. The constraint surface of the model (4.1).

\section{TWO SUBSTANCES}

Let us now consider a specific model with two substances (salt and a certain protein) $[10,11]$. Hence there are two algebraic equations $g_{1}=g_{2}=0$ which we now describe. In the following all $\beta_{i}$ are some positive constants. First we have a simple equation

$$
g_{2}=E^{2}+\beta_{1} E^{1}-\beta_{0}=0 .
$$

The map $g_{1}$ is more complicated. In general we can write it as follows:

$$
g_{1}=K_{12} \gamma_{2} C^{2}\left(\bar{\gamma}_{1} E^{1}\right)^{\beta_{2}}-\bar{\gamma}_{2} \beta_{2} E^{2}\left(\gamma_{1} C^{1}\right)^{\beta_{2}}=0 .
$$

Here $K_{12}$ is the equilibrium constant, $\gamma_{j}$ are liquid activity coefficients and $\bar{\gamma}_{j}$ are surface activity coefficients. Now we somewhat simplify the model given in $[10,11]$ by taking $\gamma_{2}=\bar{\gamma}_{1}=\bar{\gamma}_{2}=1$ and $\gamma_{1}=\exp \left(\beta_{4} C^{1}\right)$. This gives

$$
g_{1}=K_{12} C^{2}\left(E^{1}\right)^{\beta_{2}}-\beta_{2} E^{2}\left(C^{1}\right)^{\beta_{2}} \exp \left(\beta_{3} C^{1}\right)
$$

where $\beta_{3}=\beta_{2} \beta_{4}$. Now computing $\partial_{E} g$ we can check that $\operatorname{det}\left(\partial_{E} g\right)>0$ as long as values of $E$ and $C$ remain strictly positive.

Since $g_{2}$ is simply a linear equation we can eliminate the variable $E^{2}$ from the system. Let us now write the equations in this case and for simplicity of notation we denote $E=E^{1}$ and note that now $z$ is scalar. Let us further denote $\psi(E, S)=\left(E-S^{1}, \beta_{0}-\beta_{1} E-S^{2}\right)$. Then the initial system we can be written as:

$$
\begin{gathered}
C_{t}-d C_{x x}+v C_{x}+\alpha k \psi=0 \\
S_{t}-k \psi=0 \\
f(E, C)=K_{12} C^{2} E^{\beta_{2}}-\beta_{2}\left(\beta_{0}-\beta_{1} E\right)\left(C^{1}\right)^{\beta_{2}} \exp \left(\beta_{3} C^{1}\right)=0 .
\end{gathered}
$$

Here $f: \mathbb{R}^{3} \rightarrow \mathbb{R}$ and we denote $M=f^{-1}(0)$. Hence the solution should be such that it stays on the surface/manifold $M$. Note that $M$ is smooth for positive values of variables but it is singular when $E=C^{1}=0$. Thinking $M$ as a graph over $\left(C^{1}, C^{2}\right)$ plane for $C^{j}$ positive it is seen that $0<E<\beta_{0} / \beta_{1}$ (see Fig. 1). 
One can say that $M$ has 4 distinct regimes:

(i) the low plateau $M_{0}$ where $E \approx 0$;

(ii) the high plateau $M_{1}$ where $E \approx \beta_{0} / \beta_{1}$;

(iii) the slope $M_{2}$ where one rises steeply from $M_{0}$ to $M_{1}$ along $C^{1}$ direction;

(iv) the wall $M_{3}$ where $C^{2} \approx 0$.

The curvature of the surface is very big when one moves from one regime to another. One may summarize this by saying that the nonlinearities of $M$ are very strong but geometrically the shape of $M$ is quite simple.

When we specialize the equation (3.3) to the case where we have only one constraint equation it is helpful to introduce the following notations

$$
f_{E}=\frac{\partial f}{\partial E}, \quad \nabla_{C} f=\left(\frac{\partial f}{\partial C^{1}}, \frac{\partial f}{\partial C^{2}}\right), \quad f_{i j}=\frac{\partial^{2} f}{\partial C^{i} \partial C^{j}} .
$$

Note that $f_{12}=f_{22}=0$. Then we can write the new equation as

$$
\begin{aligned}
& f_{E} E_{t}-d \partial_{x}\left(f_{E} E_{x}\right)+f_{E} \hat{v} E_{x}-d f_{11}\left(C_{x}^{1}\right)^{2}-\alpha k\left\langle\nabla_{C} f, \psi\right\rangle=0 \\
& \text { where } \hat{v}=v-\frac{d\left\langle\nabla_{C} f_{E}, C_{x}\right\rangle}{f_{E}} .
\end{aligned}
$$

Hence the completed system is

$$
\begin{aligned}
& C_{t}-d C_{x x}+v C_{x}+\alpha k \psi=0, \\
& S_{t}-k \psi=0, \\
& f_{E} E_{t}-d \partial_{x}\left(f_{E} E_{x}\right)+f_{E} \hat{v} E_{x}-d f_{11}\left(C_{x}^{1}\right)^{2}-\alpha k\left\langle\nabla_{C} f, \psi\right\rangle=0, \\
& f(E, C)=0 .
\end{aligned}
$$

Let us again denote the above system simply as $A_{0} u=0$. The compatibility operator is thus

$$
A_{1}=\left(-\nabla_{C} f, 0,-1, \partial_{t}-d \partial_{x x}+v \partial_{x}\right) .
$$

This gives the augmented system

$$
\begin{aligned}
& C_{t}-d C_{x x}+v C_{x}+\alpha k \psi-\nabla_{C} f z=0, \\
& S_{t}-k \psi=0 \\
& f_{E} E_{t}-d \partial_{x}\left(f_{E} E_{x}\right)+f_{E} \hat{v} E_{x}-d f_{11}\left(C_{x}^{1}\right)^{2}-\alpha k\left\langle\nabla_{C} f, \psi\right\rangle-z=0, \\
& z_{t}-d z_{x x}-v z_{x}+f(E, C)=0 .
\end{aligned}
$$

\section{Numerical Results}

The aim of the simulations that follow is to explore three issues. First, we illustrate how to use the extra information contained in the augmented system in a given simulation program. Moreover we show how this in turn gives a much more accurate solution than the one obtained with the original program. Then we discuss the impact of our new formulation on epistemic uncertainties (related both to the modeling and to the numerical scheme). Finally we give an example of the impact on aleatory uncertainties in a situation where a parameter is uniformly distributed over an interval.

In the description of the model problem all quantities related to length are in centimeters, time is in seconds and concentrations are moles per litre. We consider a one dimensional domain of length 1 . The constants of the model are set as in $[10,11]$ :

$$
\begin{aligned}
& \alpha=1.38 \text {, } \\
& k=6.0, \\
& \beta_{0}=12.0, \\
& \beta_{1}=0.0334, \\
& \beta_{2},=4.91 \text {, } \\
& \beta_{3}=2.51 \text {, } \\
& K_{12}=1.05 \times 10^{10} \text {. }
\end{aligned}
$$




\subsection{Basic setting of computations}

Suppose we have a program solving the system (4.1). We denote by $h$ the space discretization parameter and the time step is given by $\delta t=0.2 h^{2} /(d+h|v|)$. The program uses explicit Euler scheme for the time discretization and standard finite differences for space discretization. To handle transport related stability issues the advection term has been discretized using a second order upwind scheme. This introduces a numerical viscosity which is bounded by $0.5 h|v|$. We have done the simulations with two different meshes corresponding to $h=10^{-2}$ and $h=5 \times 10^{-3}$. The velocity and the diffusion coefficients were chosen as $v=1$ and $d=0.1$. Thus for the coarser mesh the numerical viscosity is less than 0.005. Despite the fact that this is quite small compared to $d$ it appears to be necessary to use an upwind scheme to stabilize the calculations.

The initial concentrations are set to zero. Since $v>0$ we must specify the inlet values at the left boundary for $C^{1}$ and $C^{2}$. These were chosen as $C_{\text {in }}^{1}=1$ and $C_{\text {in }}^{2}=0.3$. Since $z$ should be as small as possible it has been initially set to zero and $z_{\text {in }}=0$ and $\left(z_{x}\right)_{\text {out }}=0$.

\subsection{Solution of the initial system}

We compute $z$ even for the initial model, without otherwise using its value when computing the values of other variables. In this way $z$ can be seen as an error indicator as well as an uncertainty indicator even for the initial system (see Sect. 5.4). This leads to the following discrete form of the system (4.1):

$$
\begin{aligned}
& \frac{1}{\delta t}\left(C_{h}^{n+1}-C_{h}^{n}\right)-(d+0.5 h|v|) C_{x x, h}^{n}+v C_{x, h}^{n}+\alpha k \psi_{h}^{n}=0, \\
& \frac{1}{\delta t}\left(S_{h}^{n+1}-S_{h}^{n}\right)-k \psi_{h}^{n}=0, \\
& E_{h}^{n+1} \leftarrow f\left(E_{h}^{n+1}, C_{h}^{n+1}\right)=0, \\
& \frac{1}{\delta t}\left(z_{h}^{n+1}-z_{h}^{n}\right)-d z_{x x, h}^{n}-v z_{x, h}^{n}+f\left(E_{h}^{n+1}, C_{h}^{n+1}\right)=0 .
\end{aligned}
$$

Since $E$ variable has no natural time evolution equation in the initial system we must use the algebraic equation $f(E, C)=0$ in (5.1) to compute $E$ at the next time instant. Note that efficient and accurate solution of the the algebraic equation is the main issue with model (5.1) since the time evolution of the differential equations is standard. Our aim is to show how to use our augmented model to remove this difficulty.

The original program uses Newton iterations at each time and for each mesh point to compute $E$ :

$$
\begin{gathered}
E_{h}^{n, p+1}=E_{h}^{n, p}-\left(f_{E}\left(E_{h}^{n, p}, C_{h}^{n+1}\right)\right)^{-1} f\left(E_{h}^{n, p}, C_{h}^{n+1}\right), \quad E_{h}^{n+1}=E_{h}^{n, \infty}, \\
f\left(E_{h}^{n, p}, C_{h}^{n+1}\right)=K_{12} C_{h}^{2, n+1}\left(E_{h}^{n, p}\right)^{\beta_{2}}-\beta_{2}\left(\beta_{0}-\beta_{1} E_{h}^{n, p}\right)\left(C_{h}^{1, n+1}\right)^{\beta_{2}} \exp \left(\beta_{3} C_{h}^{1, n+1}\right) .
\end{gathered}
$$

As stopping criteria it uses the value of the constraint and a bound on the number of iterations in $p$.

\subsection{Using the augmented system}

The basic idea is to take advantage of the dynamical equation for $E$ given in (4.3) and use it to provide a better initialization for the Newton iterations. Hence given $C^{n+1}, E^{n}$ and $S^{n+1}$ we first compute an intermediate value $\tilde{E}^{n}$ which we then use as an initial value for Newton iteration if $|f|>$ tol:

$$
\begin{gathered}
\tilde{E}_{h}^{n}=E_{h}^{n}+\delta t\left(f_{E, h}^{n+1 / 2}\right)^{-1}\left(d \partial_{x}\left(f_{E, h}^{n+1 / 2} E_{x, h}^{n}\right)-f_{E, h}^{n+1 / 2} \hat{v}_{h}^{n+1 / 2} E_{x, h}^{n}\right. \\
\left.+d f_{11}\left(C_{x, h}^{1, n+1}\right)^{2}+\alpha k\left\langle\nabla_{C} f_{h}^{n+1 / 2}, \psi_{h}^{n+1 / 2}\right\rangle+z_{h}^{n}\right),
\end{gathered}
$$






Figure 2. Proportion of discretization points where the Newton algorithm fails for the initial system (4.1) for the 100 and 200 (lower curve) points meshes. Iterations succeed when initialized with $E$ from (4.3).

where

$$
\begin{aligned}
& f_{h}^{n+1 / 2}=f\left(E_{h}^{n}, C_{h}^{n+1}\right), \\
& \psi_{h}^{n+1 / 2}=\left(E_{h}^{n}-S_{h}^{1, n+1}, \beta_{0}-\beta_{1} E_{h}^{n}-S_{h}^{2, n+1}\right), \\
& \hat{v}_{h}^{n+1 / 2}=v-d\left\langle\nabla_{C} f_{E, h}^{n+1 / 2}, C_{x, h}^{n+1}\right\rangle / f_{E, h}^{n+1 / 2} .
\end{aligned}
$$

But equation (5.2) involves several nonlinear expressions and brings new difficulties. Recall that $z_{h}$ can be considered as a local measure of the error. Hence neglecting the nonlinear terms would typically increase greatly the value of $\left|z_{h}\right|$. However, (5.2) is only used as an initialization to the Newton's method so we expect that $\left|z_{h}\right|$ is much reduced if the iteration converges successfully. Hence it is conceivable that one can simplify (5.2) considerably, and yet maintain the good convergence properties. We propose the following formula as a candidate for such a compromise:

$$
\tilde{E}_{h}^{n}=E_{h}^{n}+\delta t\left(d E_{x x, h}^{n}-\left(f_{E, h}^{n+1 / 2}\right)^{-1} z_{h}^{n}\right) .
$$

Indeed it turns out that with this choice the Newton iterations always succeed. In Figure 2 we show the ratio of the number of failures to the number of the discretization points at each time step for two meshes. Here by failure we mean that after the prescribed number of iterations the convergence criterion $|f| \leq$ tol was not fulfilled. To pursue the computation in these cases we used the final iteration of the Newton procedure. One can therefore deduce that in our discrete solution of the initial model the constraint $f(E, C)=0$ is far from being adequately satisfied. It is also interesting to investigate how much the Newton iteration changes the computed solution. In Figure 3 we compare the solutions obtained by using (5.3) without projection to the case where (5.3) is used to initialize the Newton iterations. It is seen that the projection is necessary in the regions where the solution changes rapidly. One remarks that the error decreases with mesh refinement only in regions where the solution is smooth. Mesh refinement will not therefore remove the need for this final projection. Actually the need for projection increases for finer meshes in area of rapid variations.

In Figure 4 the computed solutions are plotted both for the initial and augmented model. The variables $C^{1}$, $S^{1}, E$ and $z$ are shown at times $t=0.3$ and $t=0.6$ over the domain for the two meshes. Improving the solution of the constraint equation by an initialization with our new evolution equation for $E$ reduces the mesh dependency of the solution. 

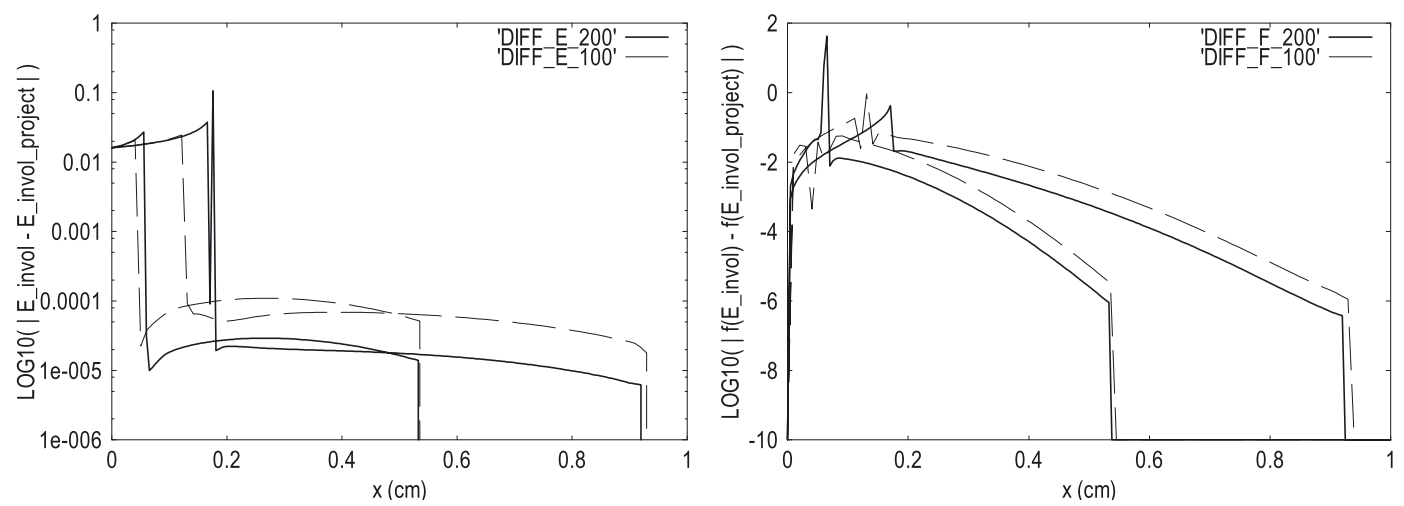

Figure 3. On the left: differences between $\tilde{E}_{h}^{n}$ given by (5.3) and its projection at times $t=0.3$ and $t=0.6$ on the meshes with 100 and 200 points. On the right: corresponding variations in $f$.

\subsection{New variable $z$ as a measure of epistemic uncertainties}

The variable $z$ is an indicator of the epistemic uncertainty both at the continuous and the discrete levels. It can be used, for instance, to measure the impact of mesh refinements on the solution when a reference solution is not available. In Figure 4 one can see that some of the deviations between the solutions of augmented and initial systems decrease with mesh refinement. However, some qualitatively incorrect features remain present in the solution obtained by the initial model such as jumps and non-monotonic behaviors. Hence, another interest of the approach is that mesh independence of the solution is achieved much faster when the constraint is better solved. The pertinence of these solution is confirmed by $z$ distributions.

\subsection{Impact on aleatory uncertainties}

Now let us see the impact of our reformulation on aleatory uncertainties. These are irreducible and related to uncertain data. Let us first consider an interval-based simulation where some of the physical parameters are given as probability distributions on an interval and not as a single value. For instance, we can consider that the transport velocity $v$ is a random variable which is uniformly distributed on the interval $[0.8,1.2]$. The initial and the augmented models have been simulated on uniform 20 and 40 points sampling of this interval on the 100 points mesh. This coincides with a classical first order numerical integration over a uniform discretization of interval $[0.8,1.2]$.

We are interested in mean values and standard deviations of the variables at different times over the domain. These quantities are of importance in robust engineering design which is often based on the control of the first and second order moments.

To see the impact of the sampling size on the numerical evaluation of the moments we consider two different samplings of size 20 and 40. In Figure 5 these cases are compared on the 100 points mesh. One sees that a 20 points discretization of the interval is nearly sufficient for an accurate numerical integration and evaluation of the two moments. We see no difference on the mean and slight overestimation of the standard deviation with the 20 points sampling. This is less problematic in robust design than an underestimation.

In Figure 6 we use 40 points sampling to compare the expected values $\mathbb{E}\left(C^{1}\right)$ and standard deviations $\left(\mathbb{E}\left(\left(C^{1}\right)^{2}\right)-\mathbb{E}\left(C^{1}\right)^{2}\right)^{1 / 2}$ of $C^{1}$ at times $t=0.3$ and $t=0.6$ for the two models for the 100 and 200 points meshes. One sees that imperfect resolution of the constraint leads to a large overestimation of the second order moment of the variable. Most importantly, the error reaches its maximum nearly where the first moments by the two methods coincide. This overestimation of the second order moment is even more visible for $E$ and $S$ in Figures 7 and 8. One also remarks that the distributions of the moments are nearly the same for the two 

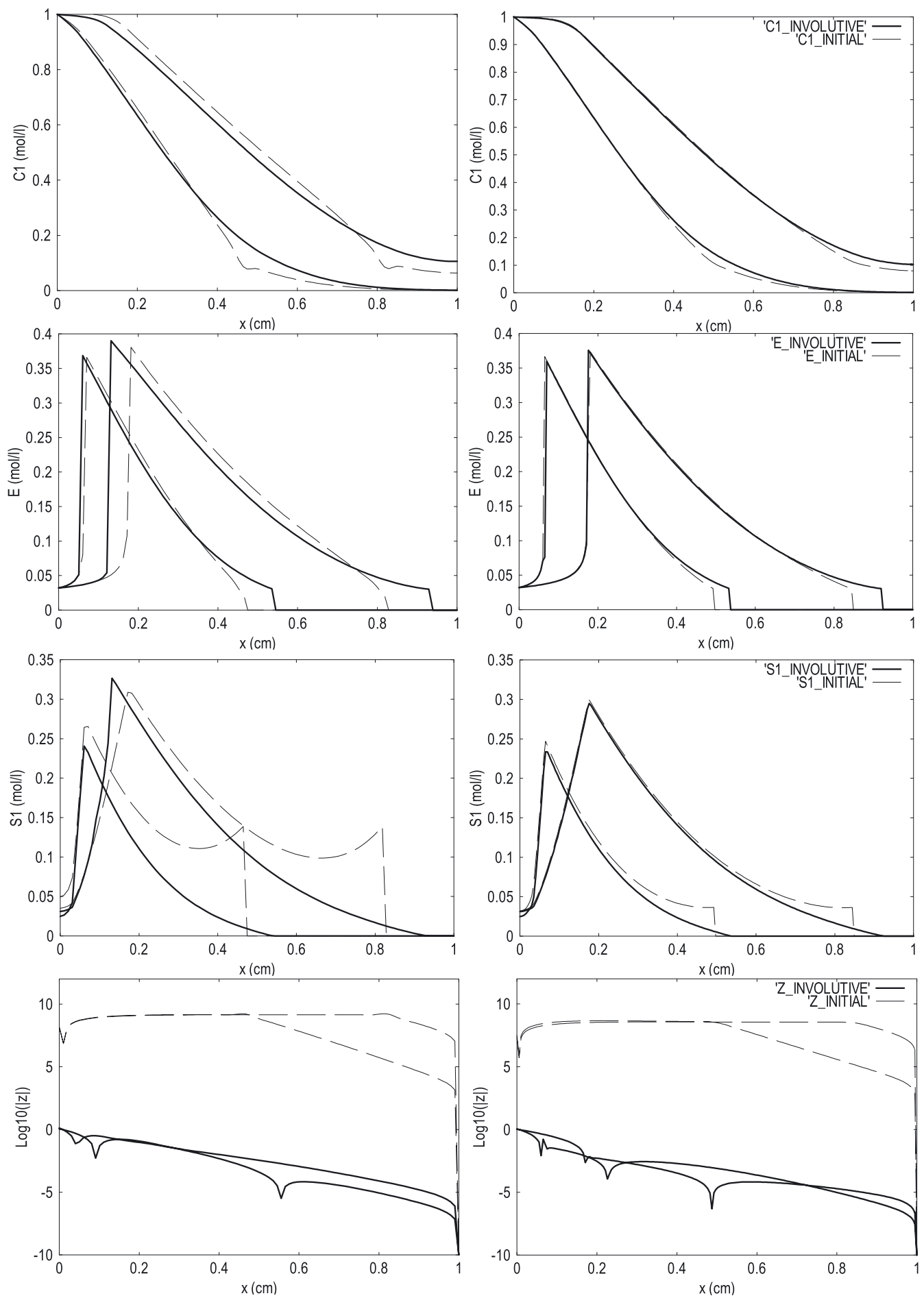

Figure 4. From the top: $C^{1}, S^{1}, E$ and $\log _{10}(|z|)$ at times $t=0.3$ and $t=0.6$ for the meshes with 100 (left column) and 200 (right column) points. 



Figure 5. Impact of the sampling size (continuous: 40, dashed: 20) on the mean value (left column) and the standard deviation (right column) for $C_{1}, E$ and $S$ at times $t=0.3$ and $t=0.6$ on the 100 points mesh for the involutive model.

meshes when the constraint is well resolved. This therefore gives more confidence on these moments since they are less sensitive to the quality of the discretization than the results obtained with the initial model.

Prediction of higher order moments and extreme values is of major importance for most engineering applications and one sees here an example of how reducing the epistemic uncertainties leads to better estimations of aleatory uncertainties.

\subsection{Propagation of perturbations from inlet boundaries}

Another classical source of aleatory uncertainty is the imprecise nature of the boundary conditions. For instance, instead of being deterministic as above, the inlet values (at the left boundary of the domain since $v>0$ ) for $C^{1}$ and $C^{2}$ can have a time dependent random component. As an example we consider the case where 

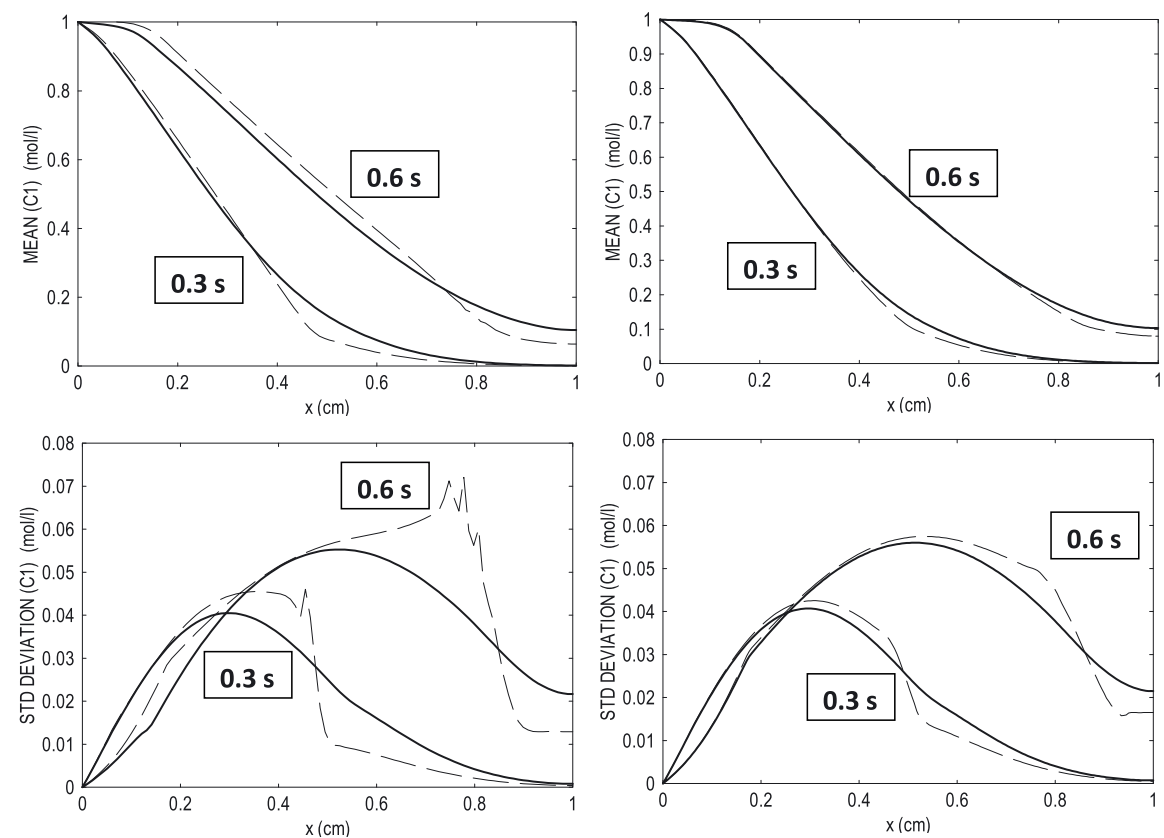

Figure 6. Mean value and standard deviation for $C_{1}$ at times $t=0.3$ and $t=0.6$ on the 100 (left column) and 200 (right column) points meshes for the involutive (continuous) and initial (dashed) models for a 40 points sampling. Extreme values of the deviation are over-estimated if the constraint is not well resolved.
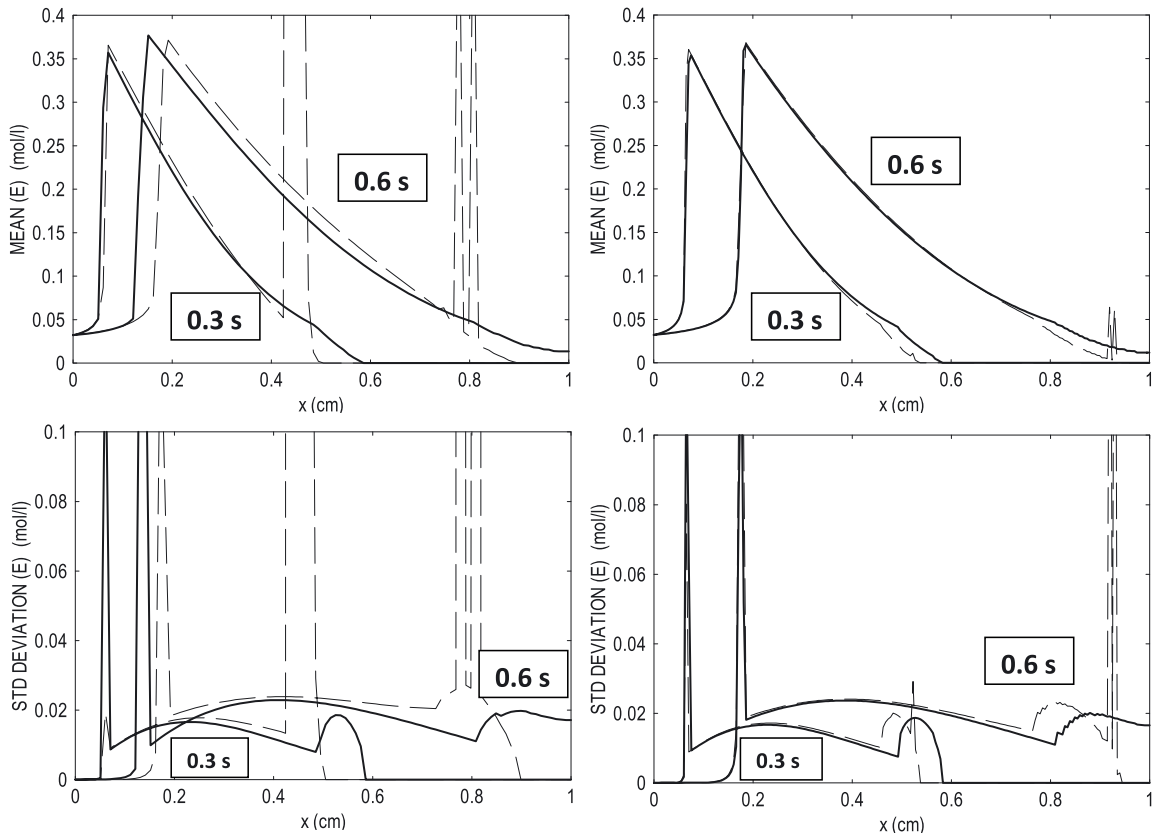

Figure 7. Mean value and standard deviation for $E$ at times $t=0.3$ and $t=0.6$ on the 100 (left column) and 200 (right column) points meshes for the involutive (continuous) and initial (dashed) for a 40 points sampling. 

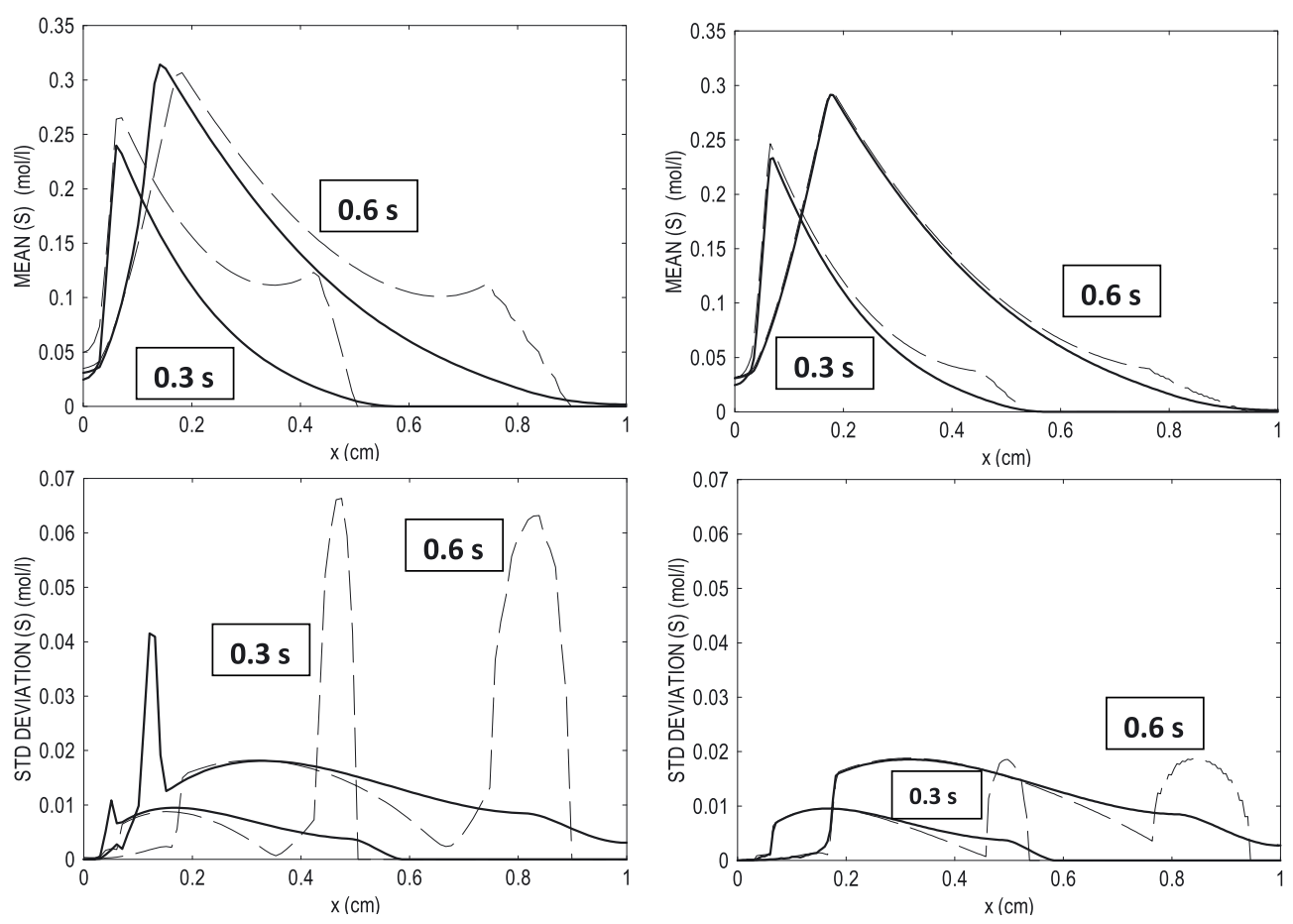

Figure 8. Mean value and standard deviation for $S$ at times $t=0.3$ and $t=0.6$ on the 100 (left column) and 200 (right column) points meshes for the involutive (continuous) and initial (dashed) for a 40 points sampling.

the inlet values are normally distributed: $C_{\mathrm{in}}^{1}=\mathcal{N}\left(1, \sigma_{1}\right)$ and $C_{\mathrm{in}}^{2}=\mathcal{N}\left(0.3, \sigma_{2}\right)$. This modeling of uncertain inlets is reasonable as the signal to noise ratio is usually known in practice. We consider here a signal to noise ratio (SNR) of $5 \%$. This introduces a high frequency component in the solution from the inlet boundary. The plane waves computed in (3.6) make us expect that the high frequency components of the augmented system are heavily damped. Therefore the uncertainties introduced through the boundary conditions would disappear when propagating in the domain. Figure 9 shows the distributions for $C_{1}, E$ and $S_{1}$ in the domain at times 0.3 and 0.6 for 20 independent simulations with the randomly perturbed boundary conditions for $C_{1}$ and $C_{2}$. We can see indeed that the perturbations do not propagate and remain very much located near the inlet left boundary. This is particularly clear for the variable $C_{1}$. A nice indication of this behavior is through $z$ profiles which, in particular, show drops where the variables $E$ and $S_{1}$ have jumps. In comparison the indicator $z$, also estimated for the initial model (see lower curves in Fig. 4), does not present any such behavior.

\section{Conclusion}

We have discussed the quantification of epistemic uncertainties in the context of numerical solution of constrained coupled systems. We have shown how our constructive algorithm for the derivation of the involutive and augmented systems provides a framework for a systematic estimation of these variabilities. The approach has been illustrated on a coupled set of partial differential, ordinary differential and algebraic equations modeling adsorption chromatography. The key idea has been to complete the system so that all variables have natural evolution equations. In particular, it has been shown that the approach naturally introduces extra variables which also play the role of epistemic uncertainty estimators. 

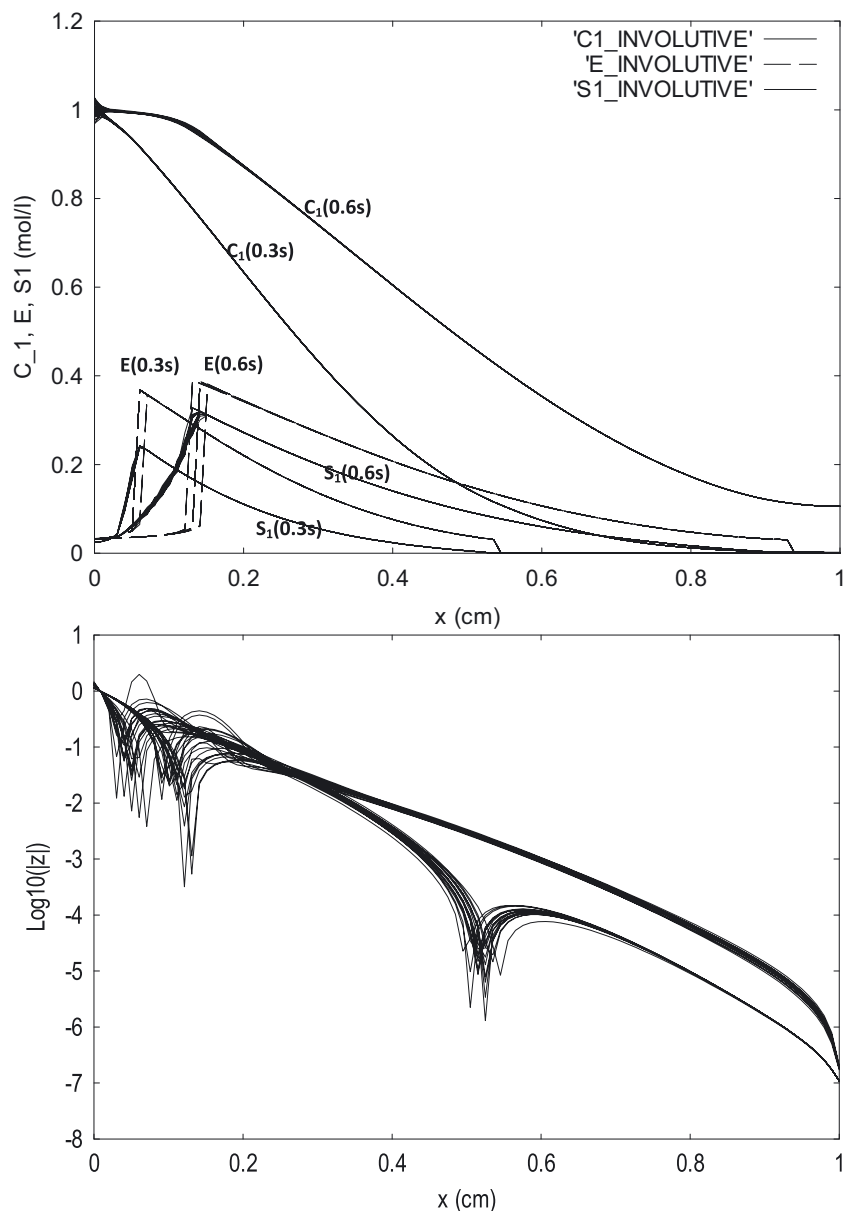

Figure 9. High frequency components of the solution $\left(C_{1}, C_{2}\right)$ introduced through the inlet boundary condition damped when propagating in the domain. Drops in $z$ appear where variables $\left(E, S_{1}\right)$ have jumps.

Working with existing software, our aim has been to introduce minimal changes in the code. It has been shown how a suitable initialization for Newton iterations used for the solution of the constraint and a control of the error through the additional variable of the augmented model improves both the robustness of the numerical scheme and the accuracy of the solution obtained.

The impact of the approach on the quantification of aleatory uncertainties has also been discussed by estimating the first and second order moments in a situation when only the probability distribution of some parameters are known. It has been shown that the prediction of the standard deviation can be quite inaccurate when the constraint in the model is not properly solved. This is of major importance in engineering applications as a robust design of a system is often based on the minimization of the mean value of a functional under a constraint on its second order moment.

Finally, the impact of uncertainties in boundary conditions has been discussed. Numerical results recover the properties predicted by the theory for the augmented model where one expects fast damping of the high frequency components of the solution introduced through small perturbations at the boundaries. This behavior is well illustrated by the indicator $z$ which clearly behaves differently for the initial and augmented systems. 
From a practical point of view, this shows that chromatographic adsorption techniques are robust with respect to small and high frequency perturbations of the species concentration and therefore cannot be used to discriminate between two samples if those are only high frequency perturbations of each other.

\section{REFERENCES}

[1] AIAA Guide for the verification and validation of computational fluid dynamics simulations. AIAA (1998) G-077.

[2] P.I. Dudnikov and S.N. Samborski, Linear overdetermined systems of partial differential equations, in Partial Differential Equations VIII, edited by M.A. Shubin. Vol. 65 of Encycl. Math. Sci. Springer (1996) 1-86.

[3] A. Ern and J.-L. Guermond, Theory and practice of finite elements. Vol. 159 of Appl. Math. Sci. Springer-Verlag, New York (2004).

[4] R. Ghanem and A. Doostan, On the construction and analysis of stochastic models: characterization and propagation of the errors associated with limited data. J. Comput. Phys. 217 (2006) 63-81.

[5] R. Ghanem and P. Spanos, Stochastic finite elements: A spectral approach. Springer Verlag, New York (1991).

[6] G. Iaccarino, Quantification of uncertainty in flow simulations using probabilistic methods. VKI Lect. Series (2008)

[7] K. Krupchyk, W. Seiler and J. Tuomela, Overdetermined elliptic systems. Found. Comp. Math. 6 (2006) 309-351.

[8] K. Krupchyk and J. Tuomela, Completion of overdetermined parabolic PDEs. J. Symb. Comput. 43 (2008) 153-167.

[9] O.P. Le Maître and O.M. Knio, Spectral methods for uncertainty quantification. Scientific Computation. Springer, New York (2010).

[10] Y. Lim, S. Chang and S. Jørgensen, A novel partial differential algebraic equation (PDAE) solver: iterative spacetime conservation element/solution element (CE/SE) method. Comput. Chem. Eng. 28 (2004) 1309-1324.

[11] Y. Lim, S. Jørgensen and I. Kim, Computer-aided model analysis for ionic strength-dependent effective charge of protein in ion-exchange chromatography. Biochem. Eng. J. 25 (2005) 125-140.

[12] B. Mohammadi and J. Tuomela, Simplifying numerical solution of constrained PDE systems through involutive completion. ESAIM: M2AN 39 (2005) 909-929.

[13] B. Mohammadi and J. Tuomela, Involutive upgrade of Navier-Stokes solvers. IJCFD 23-6 (2009) 439-447.

[14] B. Mohammadi and J. Tuomela, Involutive formulation and simulation for electroneutral microfluids. ESAIM: M2AN 45 (2011) 901-913.

[15] G. Obinata and B. Anderson, Model reduction for control system design. Springer, Berlin (2000).

[16] J. F. Pommaret, Systems of partial differential equations and Lie pseudogroups. Vol. 14 of Math. Appl. Gordon and Breach Science Publishers (1978).

[17] Z. Qu, Model order reduction techniques with applications in finite element analysis. Springer, Berlin (2004).

[18] W. Schilders, H. Van der Vorst and J. Rommes, Model order reduction: Theory, research aspects and applications. Vol. 13 of Springer Math Indus. Series. Berlin (2008).

[19] W.M. Seiler, Involution - the formal theory of differential equations and its applications in computer algebra. Vol. 24 of Algorithms Comput. Math. Springer (2010).

[20] R. Smith, Uncertainty quantification. Vol. 12 of Comput. Sci. Eng. SIAM, Philadelphia, PA (2014).

[21] D. Spencer, Overdetermined systems of linear partial differential equations. Bull. Am. Math. Soc. 75 (1969) 179-239.

[22] D. Xiu, Numerical methods for stochastic computations: A spectral method approach. Princeton University Press (2010). 